Groups Geom. Dyn. 8 (2014), 23-38

DOI $10.4171 / \mathrm{GGD} / 215$
Groups, Geometry, and Dynamics

(C) European Mathematical Society

\title{
Positive speed for high-degree automaton groups
}

\author{
Gideon Amir and Bálint Virág
}

\begin{abstract}
Mother groups are the basic building blocks for polynomial automaton groups.
We show that, in contrast with mother groups of degree 0 or 1 , any bounded, symmetric, generating random walk on the mother groups of degree at least 3 has positive speed.

The proof is based on an analysis of resistance in fractal mother graphs. We give upper bounds on resistances in these graphs, and show that infinite versions are transient.
\end{abstract}

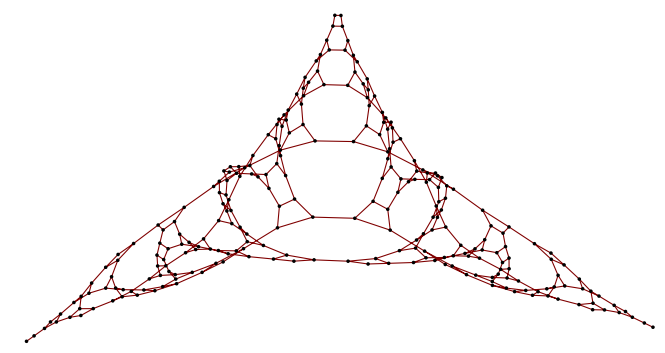

The fractal mother graph $\mathscr{H}(1,2,8)$.

Mathematics Subject Classification (2010). 60B15, 20E08, 05C21, $05 \mathrm{C} 81$.

Keywords. Automaton groups, random walks, Liouville property.

\section{Introduction}

The most interesting automaton groups, called polynomial automata, are classified according to their degree. In [10] it was shown that all these groups are small in the sense that they do not contain nonabelian free subgroups. It was asked whether all these groups were amenable, a question that is still wide open.

In [2] (for degree 0) and [1] (for all degrees) it was shown that every polynomial automaton group is contained in a mother group of the same degree. The mother groups $\mathfrak{M}_{d, n}$, defined precisely in Section 3, are a simple set of groups indexed by 
their degree $d$ and the size of the alphabet $m$. Our main theorem shows that for $d \geq 4$ or $d \geq 3, m \geq 3$ the mother groups are large in the following sense.

Theorem 1. Every bounded, generating random walk on the mother groups with $d \geq 4$ or $d \geq 3, m \geq 3$ has positive speed.

This is proved through Proposition 12 and Theorem 3. Theorem 1 is in contrast with $d=0,1$ where the mother groups support bounded, generating random walks with zero speed. ([2], [1].)

The proof of this theorem is based on the analysis of Schreier graphs of the natural action of automata groups. These graphs are discrete versions of fractals, and indeed, many classical fractals can be represented this way. Examples include the Sierpinski gasket, the long-range graph of [4], the Basilica (see [6] and [3]) and other Julia sets. The Schreier graphs $\mathcal{E}(d, m, n)$ for the mother groups, called fractal mother graphs can be described up to uniform quasi-isometry, as follows (the precise definition is given in Section 3). The vertex set is $\{0, \ldots, m-1\}^{n}$, and two vertices are connected by an edge if they differ in a single digit, and this digit is followed by at most $d+1$ nonzero digits. Figures of various fractal mother groups can be found throughout the paper.

Theorem 2. The resistance between any two vertices in $\mathcal{E}(d, m, n)$ is bounded above by

$$
\begin{array}{ll}
c\left(\frac{m}{m-1}\right)^{n} & \text { for } d=0, \\
c n^{3-d-\log _{m}(m-1)} & \text { for } d=1 \text { or } d=2, \\
c \log ^{2} n & \text { for } d=3, m=2, \\
c & \text { for } d \geq 4 \text { or } d \geq 3, m \geq 3,
\end{array}
$$

where $c$ is a constant depending on $d, m$ only.

This will be shown in Propositions 10 and 12. It is interesting to note that the graphs $\mathcal{H}(0,2, n)$ are paths, and $\mathcal{H}(0,3, n)$ are close relatives of the Sierpinski gasket, although different enough that the resistances are off by a power. See [8] for an overview of analysis on such fractals.

In an upcoming paper we plan to show a logarithmic lower bound for $d=2$ and a polynomial lower bound for $d=1$.

The graphs $\mathcal{E}(d, m, n)$ have infinite versions, defined as above with $n=\infty$. These graphs have uncountably many connected components; the notation $\mathcal{E}(d, m, \infty)$ refers to the component of the vertex ...000. In this case, we show that for $d \geq 4$ or $d \geq 3, m \geq 3$ every connected component is transient.

The second step of the proof is based on the ideas of [5]. In our setting we have the following. 


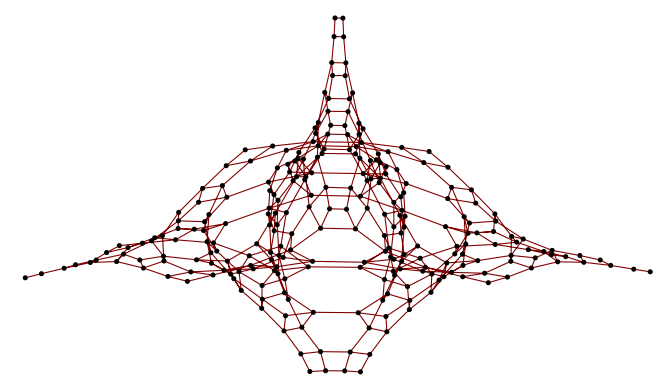

The fractal mother graph $\mathcal{E}(2,2,8)$.

Theorem 3. If $\mathscr{E}(d, m, \infty)$ is transient, then every bounded generating random walk on the group $\mathfrak{M}_{d, m}$ has positive speed.

In fact, following the proof of [5], we show that the group has nonconstant bounded harmonic functions, which is equivalent to positive speed, see [7], [11], [12] and also the lecture notes [9].

Finally, we would like to state some open questions and conjectures. The one we wish could be a theorem in this paper is

Conjecture 1. The graph $\mathcal{E}(3,2, \infty)$ is transient.

This would take care of the missing case $d=3, m=2$.

Our bounds for $d=0, d \geq 4$ and $d \geq 3, m \geq 3$ are sharp. We believe that the following is true.

Conjecture 2. The bound for $d=1$ is sharp.

In contrast, it seems that we have the following.

Conjecture 3. The maximal resistance in $\mathcal{E}(2, m, n)$ grows slower than any power of $n$.

In a future paper, we will show that $\mathcal{E}(2, m, \infty)$ is recurrent. This, however, does not decide the following

Question. Do the degree 2 mother groups support generating random walks with positive speed? Do they support generating walks with zero speed?

We have also observed, but have not been able to prove, the following. In the graph $\mathcal{E}(d, m, n)$ call the vertex $00 \ldots 00$ the root and the vertex $10 \ldots 00$ the antiroot. 
Conjecture 4. The root and the antiroot maximize the resistance in $\mathcal{E}(d, m, n)$.

Showing this would simplify our present proof.

\section{Automata and their groups}

Basic definitions. Finite automata are the simplest interesting model of computing. The space of words in alphabet $\{0, \ldots, m-1\}$ has a natural tree structure, with $\{w x\}_{x<m}$ being the children of the finite word $w$, and the empty word $\emptyset$ being the root. Let $\mathbb{T}_{m}$ denote this tree.

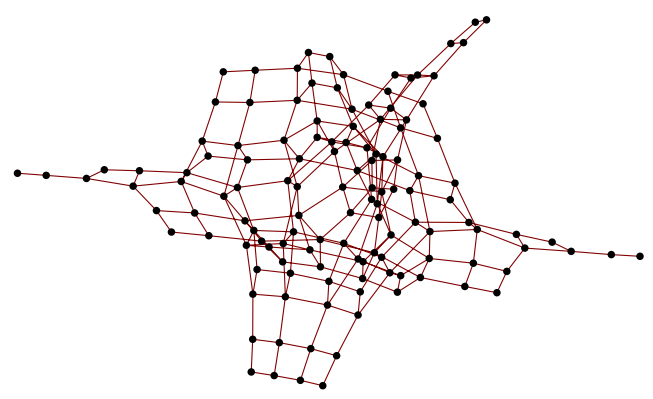

The fractal mother graph $\mathscr{U}(3,2,7)$.

A finite automaton on $m$ symbols is a finite set of states $A$ with a map $A \rightarrow$ $A^{m} \times \operatorname{Sym}(m)$ sending $a \mapsto\left(a_{0}, \ldots, a_{m-1}, \sigma_{a}\right)$. We will use the notation

$$
a=\left\langle\left\langle a_{0}, \ldots, a_{m-1}\right\rangle\right\rangle \sigma_{a} .
$$

An automaton acts on words in alphabet $\{0, \ldots, m-1\}$ sequentially. When the automaton is in a state $a$ and reads a letter $x$, it outputs $x \cdot \sigma_{a}$ and moves to state $a_{x}$. From this state the automaton acts on the rest of the word. Formally, for a word $w x$ (with the first letter from the right being the letter $x$ ) we have the recursive definition

$$
(w x) \cdot a=\left(w \cdot a_{x}\right)\left(x \cdot \sigma_{a}\right) .
$$

The first $k$ symbols of the output are determined by the first $k$ symbols read. Note that the action is defined for both finite and infinite words, and that the action on infinite words determines the action on finite words and vice versa. It follows that each element $a \in A$ is an automorphism of $\mathbb{T}_{m}$. The automaton group corresponding to an automaton $A$ is the subgroup of $\operatorname{Aut}\left(\mathbb{T}_{m}\right)$ generated by $A$.

The action (1) corresponds to the following multiplication rule:

$$
\left\langle\left\langle a_{0}, \ldots, a_{d}\right\rangle\right\rangle \sigma\left\langle\left\langle b_{0}, \ldots, b_{d}\right\rangle\right\rangle \tau=\left\langle\left\langle a_{0} b_{0 \cdot \sigma}, \ldots, a_{m-1} b_{(m-1) \cdot \sigma}\right\rangle\right\rangle \sigma \tau .
$$


This multiplication rule can be used to define automaton groups without any reference to automorphisms of the tree.

We use the conjugation notation $a^{b}=b^{-1} a b$.

The notion of first-level sections can be generalized to any level. If $v \in \mathbb{T}_{m}$ is a finite word and $g \in \operatorname{Aut}\left(\mathbb{T}_{m}\right)$, then there is a word $v^{\prime}$ of equal length to $v$ and an automorphism $g^{\prime} \in \operatorname{Aut}\left(\mathbb{T}_{m}\right)$ such that $v w \cdot g=v^{\prime}\left(w \cdot g^{\prime}\right)$, for every word $w$. This $g^{\prime}$ is called the section of $g$ at $v$. Informally, $g^{\prime}$ is the action of $g$ on the subtree above the vertex $v$. The section of $g$ at $v$ is denoted by $g(v)$.

The group Aut $\left(\mathbb{T}_{m}\right)$ also acts on infinite geodesics starting from the root, called rays; these correspond to one-sided infinite words in the alphabet. The set of rays is called the boundary $\partial \mathbb{T}_{m}$ of $\mathbb{T}_{m}$. The zero ray is the geodesic $\ldots 0000$.

Activity growth of automaton groups. For any state $a \in A$, the number of length- $n$ words $v$ such that the section $a(v)$ is not the identity satisfies a certain linear recursion. Thus this number grows either polynomially with some degree $d$ or exponentially. We define the degree of activity growth (in short, degree) of $a$ to be $d$ or $\infty$, respectively. The degree of an automaton group is the maximal degree of any of its generators. Automaton groups are said to have bounded, linear, polynomial or exponential activity growth when their degree is 0,1 , finite or infinite, respectively.

\section{Mother groups}

The mother group, denoted by $\mathfrak{M}_{d, m}$, is defined as the automaton group generated by the states

$$
\begin{aligned}
\alpha_{k, \sigma} & =\left\langle\left\langle\alpha_{k, \sigma}, \alpha_{k-1, \sigma}, 1, \ldots, 1\right\rangle\right\rangle, \quad 0 \leq k \leq d, \\
\alpha_{-1, \sigma} & =\sigma, \\
\beta_{k, \rho} & =\left\langle\left\langle\beta_{k, \rho}, \beta_{k-1, \rho}, 1, \ldots, 1\right\rangle\right\rangle, \quad 1 \leq k \leq d, \\
\beta_{0, \rho} & =\left\langle\left\langle\beta_{0, \rho}, 1, \ldots, 1\right\rangle\right\rangle,
\end{aligned}
$$

where $\sigma, \rho \in \operatorname{Sym}(m)$ are arbitrary, subject to $0 \cdot \rho=0$. The number of states in $\mathfrak{M}_{d, m}$ as defined here is $m !(d+1)+(m-1) ! d$. The same group can be generated with fewer states by taking $\sigma, \rho$ only in a minimal (2-element) set of generators of $\operatorname{Sym}(m)$ and $\operatorname{stab}(0) \subset \operatorname{Sym}(m)$ respectively. This would give a generating set of size $4 d+2$.

The actions of $\alpha_{k, \sigma}$ and $\beta_{k, \rho}$ on a word have simple descriptions. Both read the word and make no changes up to the $(k+1)$ th non-zero letter.

- If the first $k+1$ nonzero letters from the right in a word are all 1 , then $\alpha_{k, \sigma}$ permutes the next letter by $\sigma$. Otherwise it does nothing.

- If the first $k$ nonzero letters from the right in a word are all 1 , then $\beta_{k, \rho}$ permutes the next nonzero letter by $\rho$. Otherwise it does nothing. 
Thus both affect only the $(k+1)$ th non-zero letter and the letter immediately following it.

Theorem 4 (Mother groups contain all, [1]). Every degree-d automaton group is isomorphic to a subgroup of $\mathfrak{M}_{d, m}$ for some $m$.

Note that $m$ is generally not the same as the degree of the tree on which the automaton acts.

Level subgroups. Observe that the group of automorphisms of the first two levels of $\mathbb{T}_{m}$ fixing 0 and its children is isomorphic to $\operatorname{Sym}(m)<\operatorname{Sym}(m-1)$. (We will interpret elements in $\operatorname{Sym}(m-1)$ as acting on $\{1, \ldots, m-1\}$.)

For each $\sigma \in \operatorname{Sym}(m)\{\operatorname{Sym}(m-1)$ and each word $w$ in the symbols $\{1, \ldots, m-1\}$ let $\lambda_{w, \sigma}$ denote the element of Aut $\left(\mathbb{T}_{m}\right)$ acting as follows: If the first $|w|$ nonzero letters from the right agree with $w$, then $\lambda_{w, \sigma}$ permutes the $|w|+1$ st nonzero letter and the following letter by $\sigma$. Otherwise $\lambda_{w, \sigma}$ does nothing. For example, for $\sigma=(\mathrm{id},(01)) 2(12)$ we have $\ldots 002020010 \cdot \lambda_{21, \sigma}=\ldots 011020010, \ldots 001020010$. $\lambda_{21, \sigma}=\ldots 002020010$ and $\ldots 002010010 \cdot \lambda_{21, \sigma}=\ldots 002010010$.

For a word $w$ of length $k$, define the group $\mathfrak{R}_{m, k}^{w}$ generated by $\lambda_{w, \sigma}$ as $\sigma$ ranges over $\operatorname{Sym}(m)$ ? $\operatorname{Sym}(m-1)$. Define the group $\mathfrak{L}_{k, m}$ to be the group generated by the $\mathfrak{R}_{k, m}^{w}$ for all words $w$ of length $k$. Define further $\mathfrak{L}_{-1, m}=\operatorname{Sym}(m)$.

Later, we will consider random walks on the mother group whose step distribution is a convex combination of uniform measures on the subgroups $\mathfrak{L}_{k, m}$ for various $k$ 's.

Lemma 5 ([1]). For each $w, \mathfrak{R}_{k, m}^{w} \approx \operatorname{Sym}(m)>\operatorname{Sym}(m-1)$. The group $\mathfrak{R}_{k, m}$ is a subgroup of $\mathfrak{M}_{k, m}$ and is the direct product of $\mathfrak{R}_{k, m}^{w}$ for $w \in\{1, \ldots, m-1\}^{k}$. Moreover, the mother group $\mathfrak{M}_{k, m}$ is generated by the subgroups $\left\{\mathfrak{R}_{m, \ell}\right\}_{\ell \leq k}$.

Definition 6. The fractal mother graphs $\mathcal{E}(d, m, n)$ are the Schreier graphs of the mother group $\mathfrak{M}_{d, m}$ acting on level $n$ of $\mathbb{T}_{m}$ with the generating set $\bigcup_{k \leq d} \mathfrak{L}_{k, m}$ (counting multiplicity). These are regular graphs with vertex degree

$$
\operatorname{deg}(v)=\sum_{k=-1}^{d}\left|\mathfrak{L}_{k, m}\right|=m !+\sum_{k=0}^{d}(m-1)^{k}(m-1) !(m !)^{m-1}
$$

$\mathcal{E}(d, m, \infty)$ is the connected component of the zero ray of the Schreier graph of the action of $\mathfrak{M}_{d, m}$ on the boundary. The figures in this paper depict various instances of $\mathcal{E}(d, m, n)$.

\section{Basic resistance properties of fractal mother graphs}

The vertices of $\mathcal{E}(d, m, n)$ are the set of words in $\{0, \ldots, m-1\}^{n}$. There is a natural embedding $\mathcal{E}(d, m, 1) \subset \mathcal{H}(d, m, 2) \subset \cdots \subset \mathcal{E}(d, m, \infty)$ of the vertex sets of these 
graphs, given by adding zeros to the beginning of words. This embedding preserves the graph structure in the sense that the graph induced by the vertices of $\mathscr{E}(d, m, i)$ in $\mathcal{E}(d, m, i+1)$ is isomorphic to $\mathcal{E}(d, m, i)$ with some loops erased.

We introduce some notation: The $n$-digit vertices $o=00 \ldots 00$ and $\bar{o}_{n}(x)=$ $x 0 \ldots 00(x \in\{1,2, \cdot, m-1\})$ are called the root and $x$-antiroot of $\mathcal{G}(d, m, n) . \bar{o}_{n}(1)$ is called simply the antiroot of $\mathcal{E}(d, m, n)$ and denoted by $\bar{o}_{n}$. For $w \in \mathcal{E}(d, m, n)$ let \#w denote the number of nonzero letters in $w$. Finally, let $\Re_{d, m, n}(a, b)$ denote the resistance in $\mathcal{E}(d, m, n)$ between vertices or vertex sets $a, b$.

Proposition 7. (a) For $x \in \mathcal{E}(d, m, n)$ we have

$$
\Re_{d, m, n}(o, x) \leq \sum_{s: x_{s} \neq 0} \Re_{d, m, s}\left(o, \bar{o}_{s}\right) .
$$

(b) For $x, y \in \mathscr{E}(d, m, n)$ we have

$$
\Re_{d, m, n}(x, y) \leq \sum_{s=1}^{n}\left(\mathbf{1}\left(x_{s} \neq 0\right)+\mathbf{1}\left(y_{s} \neq 0\right)\right) \Re_{d, m, s}\left(o, \bar{o}_{s}\right) .
$$

Proof. (a): Let $x=x_{n} \ldots x_{1}$, and $z_{s}=x_{n} \ldots x_{s}$ followed by $s-1$ zeros. The triangle inequality for resistances gives

$$
\Re_{d, m, n}(o, x) \leq \sum_{s=1}^{n} \Re_{d, m, n}\left(z_{s-1}, z_{s}\right) .
$$

The terms where $x_{s}=0$ are 0 . To complete the proof, we need to show that

$$
\Re_{d, m, n}\left(z_{s-1}, z_{s}\right) \leq \Re_{d, m, s}\left(o, \bar{o}_{s}\right) .
$$

This is because the map (mapping words of length $s$ to words of length $n$ ) that sends a word $v$ to $x_{n} \ldots x_{k-1} v$ is an injection of vertices and extends to an injection of edges (apart from loops). Moreover,

$$
o \mapsto z_{s} \quad \text { and } \quad \bar{o}_{\ell}\left(x_{s}\right) \mapsto z_{s-1} .
$$

Thus by Rayleigh's monotonicity we get

$$
\Re_{d, m, n}\left(z_{s-1}, z_{s}\right) \leq \Re_{d, m, s}\left(o, \bar{o}_{s}\left(x_{s}\right)\right)
$$

and the right-hand side equals $\Re_{d, m, s}\left(o, \bar{o}_{s}\right)$ by symmetry.

(b) This follows from part (a) and the triangle inequality for resistances.

Let

$$
\bar{r}(d, m, n)=\max _{x, y \in \mathcal{E}(d, m, n)} \Re_{d, m, n}(x, y) .
$$


Proposition 8. (a) For $n^{\prime} \leq n$ and $x, y<m^{n^{\prime}}$ we have $\Re_{d, m, n}(x, y) \leq \Re_{d, m, n^{\prime}}(x, y)$.

(b) There exists a constant $c$ depending on $m, d$ only so that for $k \geq 1$ we have $\bar{r}(d, m, n+k) \leq c^{k} \bar{r}(d, m, n)$.

Proof. (a): This follows from Rayley's monotonicity principle and the fact that, apart from loops, $\mathscr{E}\left(d, m, n^{\prime}\right)$ is a subgraph of $\mathscr{E}(d, m, n)$.

(b): It suffices to prove this for $k=1$, the rest follow by induction. Consider the vertices $x<m^{n}$. Fix a letter $b$. Note that $x \mapsto b x$ is a graph homomorphism (except for loops). In particular, by Rayleigh's monotonicity principle for $x, y<m^{n}$ we have

$$
\Re_{d, m, n+1}(b x, b y) \leq \Re_{d, m, n}(x, y) .
$$

Recall that $\bar{o}_{n}$ is the antiroot $100 \ldots 000$ in $\mathcal{E}(d, m, n)$. For letters $a, b$ the triangle inequality for resistances implies

$$
\begin{aligned}
& \Re_{d, m, n+1}(a x, b y) \\
& \quad \leq \Re_{d, m, n+1}\left(a x, a \bar{o}_{n}\right)+\Re_{d, m, n+1}\left(a \bar{o}_{n}, b \bar{o}_{n}\right)+\Re_{d, m, n+1}\left(b \bar{o}_{n}, b y\right) .
\end{aligned}
$$

There is an edge between $a \bar{o}_{n}$ and $b \bar{o}_{n}$. So by (2) we have the upper bound

$$
\Re_{d, m, n+1}(a x, b y) \leq \Re_{d, m, n}\left(x, \bar{o}_{n}\right)+1+\Re_{d, m, n}\left(y, \bar{o}_{n}\right) \leq(2+c) \bar{r}(d, m, n) .
$$

\section{Flow construction}

The goal of this section is to give an upper bound on the maximal resistance in $\mathcal{E}(d, m, n)$. We include the proof of the following simple fact for completeness.

Lemma 9. We have $\bar{r}(0, m, n) \leq 2^{n}-1$.

Proof. It suffice to prove that there is a path of length $2^{n}-1$ to any $n$-digit number $\ell$. By the symmetry of the mother group, it suffices to show this for numbers containing the digits 0,1 only; in particular, it suffices to show this for the $m=2$ mother group. For this, for the binary string $x=x_{n} \ldots x_{1}$ consider the inverse Gray code representation $y=y_{n} \ldots y_{1}$ with bits $y_{k}=x_{n}+\cdots+x_{k}$. By checking how the two generators act on the $y$, one sees that graph indexed by the $y$ 's is the simple path $0,1,2, \ldots 2^{n}-1$ (with loops attached at the endpoints).

Proposition 10. We have $c\left(\frac{m}{m-1}\right)^{n} \leq \bar{r}(0, m, n) \leq c^{\prime}\left(\frac{m}{m-1}\right)^{n}$, where $c$, $c^{\prime}$ depend on $m$ only.

Proof. First let $d$ be general, and consider the current flow from $o$ to the set of antiroots $\bar{O}_{n}=\left\{\bar{o}_{n}(1), \ldots \bar{o}_{n}(m-1)\right\}$ in $\mathcal{E}(d, m, n)$. Note that the graph, $o$ and $\bar{O}_{n}$ are symmetric under the action of $\operatorname{Sym}(m-1)^{n}$ which changes the nonzero letters 


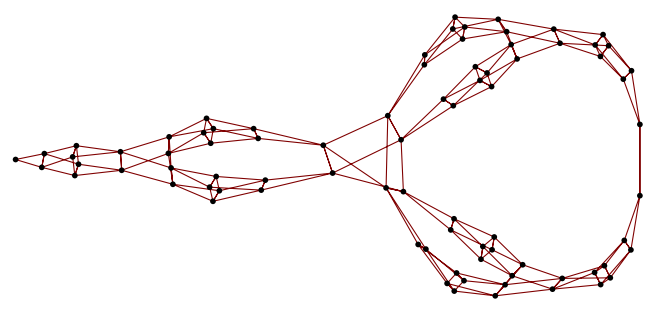

The fractal mother graph $\mathcal{E}(0,3,4)$.

of the strings of each vertex. Since the current flow is unique, it must be invariant under this action. In particular, if we identify vertices of the graph that are in the same orbit, the resistances will not change (normally, they could only decrease by Raleigh's monotonicity). After this identification, we get a weighted version of $\mathcal{E}(d, 2, n)$ with vertices given by binary strings. Each vertex $y$ is the image of $(m-1)^{\# y}$ vertices in $\mathcal{E}(d, 2, n)$. For $d=0$ this is graph is a simple path (Lemma 9), and the resistance between the endpoint can be computed explicitly. Here we estimate it by a quick argument: each vertex has one or two incident edges. The weight of these edges is within constants of the weight of the vertex. By the series formula, the resistance is given by the sum over the edges of $w(e)^{-1}$, which is therefore within constants of the sum over the vertices of $w(v)^{-1}$, that is

$$
\sum_{v=0}^{2^{n}-1}(m-1)^{-\# v}=\left(\frac{m}{m-1}\right)^{n}
$$

computed via the binomial formula. Finally, note that symmetry implies

$$
\Re_{d, m, n}\left(o, \bar{O}_{n}\right) \leq \Re_{d, m, n}\left(o, \bar{o}_{n}\right) \leq(m-1)^{2} \Re_{d, m, n}\left(o, \bar{O}_{n}\right)
$$

for the last inequality, note that the current flow to $O_{n}$ has strength $1 /(m-1)$ going into $\bar{o}_{n}$, so the energy of $(m-1)$ times this flow is an upper bound for the resistance between $o$ and $\bar{o}_{n}$. Finally, Proposition 7 (b) implies the bound on $\bar{r}$.

One way to get a transient graph is to construct a product structure; for example $\mathbb{Z} \times \mathbb{Z} \times \mathbb{Z}$ is transient. We would like to construct something that resembles the product of the Schreier graphs of a degree $d$ group and a degree $d^{\prime}$ group. It will turn out to be a subgraph of the zero-ray Schreier graph of the mother group of degree $d$ for greater $n$.

Recall that $\bar{r}(d, m, n)$ denotes the maximal resistance between vertices in $\mathcal{E}(d, m, n)$. The core of the argument is contained in the following proposition. 
Proposition 11. For any nondecreasing sequence $\gamma_{s} \geq 1$ and $n \geq 3$ we have

$$
\bar{r}(d, m, n) \leq c \sum_{s=1}^{n-2}\left[\frac{\bar{r}\left(d^{\prime}, m,\left\lfloor\gamma_{s}\right\rfloor\right)}{s^{d-d^{\prime}-1}}+\frac{\bar{r}(d, m, s)}{m^{\gamma_{s}}}\right],
$$

where $0 \leq d^{\prime} \leq d-1$ is an integer and $c$ is a constant depending on $m$ and $d$ only.

Proof. We first assume that the $\gamma_{s}$ are integers, $\gamma_{1}=\cdots=\gamma_{d}=1$, and $n \geq d+2$. Let $a, a^{\prime}$ be two vertices in $\mathcal{E}(d, m, n)$. Thompson's principle says that resistance between two vertices equals the energy of the minimal energy unit flow between them. We will construct a unit flow from $a$ to $a^{\prime}$ which has the right bounds. We will construct this flow as a sum of many parts.

Let $a_{k}$ denote $a$ with its lowest $k$ digits erased. Let $a_{k}^{*}$ denote $a_{k}$ with its lowest digit permuted cyclically by $(0 \ldots m-1)$. Let $\sigma$ be the smallest number so that $\gamma_{\sigma}+1+\sigma \geq n$; we note that $\sigma \geq d$.

Recall that \#y denotes the number of nonzero digits of $y$. For $d-1 \leq s<\sigma$ let

$$
X_{s}=\left\{a_{\gamma_{s}+s+1}^{*} x: 0 \leq x<m^{\gamma_{s}}\right\},
$$

where $a_{\gamma_{s}+s+1}^{*} x$ denotes the concatenation of $a_{\gamma_{s}+s+1}^{*}$ and the $\gamma_{s}$-digit version of $x$. Let

$$
Y_{s}=\left\{0 \leq y<m^{s}: \# y=d-d^{\prime}-1\right\} .
$$

We will think of numbers in $Y_{s}$ as written in their base- $m, s$-digit form. Finally, define

$$
X_{\sigma}=\left\{x: 0 \leq x<m^{n-\sigma}\right\} .
$$

For $x \in X_{s}$ and $y \in Y_{s}$ we denote by $x 1 y$ the obvious concatenation, and let $X_{t} 1 Y_{s}$ denote the set of such concatenations; let $X_{t} 01 Y_{s}$ be defined similarly.

We now consider flows

$$
\begin{aligned}
a \rightarrow X_{d-1} 1 Y_{d-1} \rightarrow X_{d} 01 Y_{d-1} \rightarrow & X_{d} 1 Y_{d} \rightarrow X_{d+1} 01 Y_{d} \\
& \rightarrow X_{d+1} 1 Y_{d+1} \rightarrow \cdots \rightarrow X_{\sigma} 01 Y_{\sigma-1},
\end{aligned}
$$

each transporting uniform measure from one set to uniform measure on the next. These vertex sets and flows will not be disjoint, but we will be able to control overlaps.

Let $\phi$ be the sum of these flows. Let $\phi^{\prime}$ be the sum of the same flows constructed for $a^{\prime}$, and let $\bar{\phi}$ be its reversal. Then $\phi+\bar{\phi}$ is a unit flow from $a$ to $a^{\prime}$.

Let $\varepsilon(\phi)$ denote the energy of the flow $\phi$, i.e., $\sum_{e} \phi(e)^{2}$. To bound the energy of $\phi$, we first bound the energy of its components; these alternate between two types.

Horizontal spread $X_{s-1} 1 Y_{s-1}$ to $X_{s} 01 Y_{s-1}$. Fix $y \in Y_{s-1}$. Consider edges of the form $\left(a_{s+\gamma_{s}+2}^{*} x 1 y, a_{s+\gamma_{s}+2}^{*}(g . x) 1 y\right)$ where $g$ is a generator of degree at most $d^{\prime}$ and $x, g . x<m^{\gamma_{s}+2}$. The subgraph of $\mathcal{E}(d, m, n)$ spanned by these edges is isomorphic (up to loops) to $\mathcal{E}\left(d^{\prime}, m, \gamma_{s}+2\right)$ through the map $x \mapsto a_{s+\gamma_{s}+2}^{*} x 1 y$. 
We call such edges horizontal. By Thompson's principle for any $x_{1} \in X_{s-1}$ and $x_{2} \in X_{s}$ there exists a unit flow $\phi_{s, x_{1}, x_{2}, y}^{h}$ from $x_{1} 1 y$ to $x_{2} 01 y$ to along these edges satisfying

$$
\mathcal{E}\left(\phi_{s, x_{1}, x_{2}, y}^{h}\right) \leq \bar{r}\left(d^{\prime}, m, \gamma_{s}+2\right) .
$$

Let $\phi_{s, y}^{h}$ denote the average of these flows over all $x_{1} \in X_{s-1}, x_{2} \in X_{s}$. By convexity of energy, we have

$$
\mathcal{E}\left(\phi_{s, y}^{h}\right) \leq \bar{r}\left(d^{\prime}, m, \gamma_{s}+2\right) .
$$

We now average these flows over all $y \in Y_{s-1}$ to get the flow $\phi_{s}^{h}$. Since these flows have disjoint support, and since $\left|Y_{s-1}\right| \geq c s^{d-d^{\prime}-1}$ we get that

$$
\mathcal{E}\left(\phi_{s}^{h}\right) \leq c s^{1+d^{\prime}-d} \bar{r}\left(d^{\prime}, m, \gamma_{s}+2\right) \leq c s^{1+d^{\prime}-d} \bar{r}\left(d^{\prime}, m, \gamma_{s}\right) .
$$

The last inequality follows from Proposition 8 (a). The flow $\phi_{s}^{h}$ transports uniform measure form $X_{s-1} 1 Y_{s-1}$ to uniform measure on $X_{s} 01 Y_{s-1}$.

A trivial modification of the argument gives a flow $\phi_{\sigma}$ from $X_{\sigma-1} 1 Y_{\sigma-1}$ to $X_{\sigma} 01 Y_{\sigma-1}$ with energy bounded by

$$
\mathcal{E}\left(\phi_{\sigma}^{h}\right) \leq c \sigma^{1+d^{\prime}-d} \bar{r}\left(d^{\prime}, m, \gamma_{\sigma}\right)
$$

Vertical spread, $X_{s} 01 Y_{s-1}$ to $X_{s} 1 Y_{s}$. Fix $x \in X_{s}$. Consider edges of the form $\left(x y_{s}, x g \cdot y\right)$ where $g$ is a generator of degree at most $d$, and $y, g \cdot y<m^{s+1}$ and $y, g \cdot y$ above are in their $(s+1)$-digit form. The subgraph of $\mathcal{E}(d, m, n)$ spanned by these edges is isomorphic (up to loops) to $\mathscr{E}(d, m, s+1)$. We call such edges vertical. By Thompson's principle for any $y_{1} \in Y_{s-1}$ and $y_{2} \in Y_{s}$ there exists a unit flow $\phi_{s, x, y_{1}, y_{2}}^{v}$ from $x 01 y_{1}$ to $x 1 y_{2}$ along these edges satisfying

$$
\mathcal{E}\left(\phi_{s, x, y_{1}, y_{2}}^{v}\right)=\Re_{d, m, s+1}\left(01 y_{1}, 1 y_{2}\right) .
$$

Let $\phi_{s, x}^{v}$ denote the average of these flows over all $y_{1} \in Y_{s-1}$ and $y_{2} \in Y_{s}$. By convexity of energy, we have

$$
\mathcal{E}\left(\phi_{s, x}^{v}\right) \leq \bar{r}(d, m, s+1) .
$$

We now average these flows over all $x \in X_{s}$ to get the flow $\phi_{s}^{v}$. Since these flows have disjoint support, we get that

$$
\mathcal{E}\left(\phi_{s}^{h}\right) \leq \frac{1}{m^{\gamma_{s}}} \bar{r}(d, m, s+1) \leq \frac{c}{m^{\gamma_{s}}} \bar{r}(d, m, s) .
$$

The last inequality follows from Proposition 8 (a). The flow $\phi_{s}^{v}$ transports uniform measure on $X_{s} 01 Y_{s-1}$ to uniform measure on $X_{s} 1 Y_{s}$.

We consider the flow from $o$ to $V_{4, s}$ constructed piecewise as above, namely

$$
\phi=\phi_{a}+\phi_{d}^{h}+\phi_{d}^{v}+\cdots+\phi_{\sigma-1}^{v}+\phi_{\sigma}^{h} .
$$


Here $\phi_{a}$ a unit current flow from $a$ to uniform measure on $X_{d-1} 1 Y_{d-1}$, and its energy is bounded by some constant.

We first note that the flows $\phi_{s}^{h}$ for different $s<\sigma$ are vertex-disjoint. This is because these flows move between vertices of the form $x 1 y$, with $y \in Y_{s-1}$ fixed. As long as one can determine $s$ from $x 1 y$, the vertex-disjointness follows. But since $\# y=d-d^{\prime}-1$, the $\left(d^{\prime}+2\right)$ th nonzero digit from the right in $x 1 y$ is in position $s$.

Similarly, we show that the flows $\phi_{s}^{v}$ are vertex disjoint for different $s<\sigma$. Again, these flows are vertex-disjoint. They move between vertices of the form $x y$ with $y<m^{s+1}$ is in $s+1$-digit form, and $x \in X_{s}$ is fixed. This is why we permuted a bit in $a^{*}$ : the highest digit in $x y$ that differs from the digit at the same position in $a$ is exactly at position $s+\gamma_{s}+2$. Since the map $s \mapsto s+\gamma_{s}$ is strictly increasing, the value of $s+\gamma_{s}$ determines the value of $s$.

Note that the energy of the sum of flows $\sum \phi_{i}$ is bounded above by $\sum m_{i} \&\left(\phi_{i}\right)$ where $m_{i}$ is the number of flows (including itself) that the flow $\phi_{i}$ shares an edge with. Breaking up the flow $\phi$ into four parts, namely the sum of the horizontal flow terms, the vertical terms, and $\phi_{a}$ as well as $\phi_{\sigma}$,

$$
\mathcal{E}(\phi) \leq 4 \mathcal{E}\left(\phi_{a}\right)+4 c \sum_{s=d}^{\sigma} \frac{\bar{r}\left(d^{\prime}, m, \gamma_{s}\right)}{s^{d-d^{\prime}-1}}+4 c \sum_{s=d}^{\sigma-1} \frac{\bar{r}(d, m, s)}{m^{\gamma_{s}}} .
$$

Note that the flow $\phi_{a}$ can be chosen so that its energy is bounded by a constant depending on $d, m$ only. We can remove the additive constants by increasing the multiplicative one and using that $r\left(d^{\prime}, m, \cdot\right)$ is bounded below. Then Thompson's principle gives

$$
\begin{aligned}
\Re_{d, m, n}\left(a, a^{\prime}\right) \leq \mathcal{E}(\phi+\bar{\phi}) & \leq 2 \mathcal{E}(\phi)+2 \mathcal{E}(\bar{\phi}) \\
& \leq c \sum_{s=d}^{\sigma} \frac{\bar{r}\left(d^{\prime}, m, \gamma_{s}\right)}{s^{d-d^{\prime}-1}}+c \sum_{s=d}^{\sigma-1} \frac{\bar{r}(d, m, s)}{m^{\gamma_{s}}} .
\end{aligned}
$$

Since $\sigma \leq n-2$, the inequality (3) follows for $\gamma_{s}, n$ satisfying the assumptions at the beginning of the proof. Using the fact that $\bar{r}\left(d^{\prime}, m, \cdot\right)$ is bounded below, and Proposition 8 (b) it is straightforward to see that (3) holds with a modified constant $c$ for general $\gamma_{s}, n$.

The proof of the following standard fact is a simple exercise.

Fact. Let $G$ be a bounded degree graph with a vertex o with an infinite sequence of vertices whose resistance to o is bounded. Then $G$ is transient.

Proposition 12. For $d \geq 1, m \geq 2$ we have

$$
\bar{r}(d, m, n) \leq \begin{cases}c & \text { for }\{d \geq 4, m \geq 2\} \text { or }\{d \geq 3, m \geq 3\}, \\ c \log ^{2} n & \text { for }\{d=3, m=2\}, \\ c s^{3-d-\log _{m}(m-1)} & \text { for }\{1 \leq d \leq 2\},\end{cases}
$$


where $c$ depends on $m, d$ only. In particular, in the first case, $\mathscr{E}(d, m, \infty)$ is transient. Proof. Set $d^{\prime}=0$, and set

$$
r(s)=r(d, m, s)=\max _{1 \leq \ell \leq s} \bar{r}(d, m, \ell)
$$

to guarantee that $r$ is nondecreasing. Proposition 11 together with Lemma 9 gives

$$
r(n+2) \leq c \sum_{s=1}^{n} \frac{\left(\frac{m}{m-1}\right)^{\gamma_{s}}}{s^{d-1}}+\frac{r(s)}{m^{\gamma_{s}}} .
$$

Since we are free to choose $\gamma_{s}$, we will find the approximate minimizer of the righthand side. When the two terms are equal, we are off from the minimum by at most a factor of two. The calculation gives the choice

$$
\gamma_{s}=\log _{m^{2} /(m-1)}\left(r(s) s^{d-1}\right)
$$

which is indeed nondecreasing and gives

$$
r(n+2) \leq c \sum_{s=1}^{n} \frac{r(s)^{1-\eta}}{s^{(d-1) \eta}}, \quad \eta=\left(2-\log _{m}(m-1)\right)^{-1} .
$$

Let $f(s)$ denote the summand on the right-hand side. We extend $r$ to a piecewise linear continuous function on $[1, \infty)$. For this function we have

$$
r(t) \leq r(\lceil t\rceil) \leq \sum_{s=1}^{\lceil t\rceil-2} f(s) d s \leq \int_{1}^{\lceil t\rceil-1} f(s) d s \leq \int_{1}^{t} f(s) d s
$$

for $t \geq 3$. In particular, adding a constant, we can make the inequality valid for all $t \geq 1$ :

$$
r(t) \leq c+c \int_{1}^{t} \frac{r(s)^{1-\eta}}{s^{(d-1) \eta}} d s .
$$

Consider the solution of the differential equation

$$
u^{\prime}(s)=\kappa \frac{u(s)^{1-\eta}}{s^{(d-1) \eta}}
$$

given by

$$
u(s)= \begin{cases}\left(\frac{\kappa \eta\left(1-s^{1-(d-1) \eta}\right)}{(d-1) \eta-1}\right)^{\frac{1}{\eta}} & \text { for }(d-1) \eta>1, \\ (\eta \kappa \log (s))^{\frac{1}{\eta}} & \text { for }(d-1) \eta=1, \\ \left(\frac{\kappa \eta s^{1-(d-1) \eta}}{1-(d-1) \eta}\right)^{\frac{1}{\eta}} & \text { for }(d-1) \eta<1 .\end{cases}
$$


For $\kappa$ large enough (and greater than the constant in front of the integrand in (4)) we have that $u(2)$ will be greater than the right-hand side of (4) evaluated at $s=2$. For such $\kappa$ we claim that $u(t)>r(t)$ for all $t \geq 2$. Assume the contrary; since $r, u$ are continuous, then there is a smallest $t \geq 2$ so that $u(t)=r(t)$. Therefore

$$
r(t) \leq c+c \int_{1}^{t} \frac{r(s)^{1-\eta}}{s^{(d-1) \eta}} d s<u(2)+\kappa \int_{2}^{t} \frac{u(s)^{1-\eta}}{s^{(d-1) \eta}} d s=u(t),
$$

a contradiction. In terms of $d$ and $m$, the inequality deciding the cases above is $d \geq 3-\log _{m}(m-1)$. The claim of the corollary follows.

\section{From transience to nontrivial Poisson boundary}

Let $K$ denote the subgroup of $\mathfrak{M}_{d, m}$ generated by all generators except those of degree -1 . Note that all elements of $K$ fix the zero ray. Let $H$ denote the subgroup of $K$ generated by all generators excepts those of degree -1 or degree $d$.

Proposition 13. For every $g$ in the mother group, as $n \rightarrow \infty$ the $n$th level section at the zero ray is eventually constant and is in $K$. We call this $\operatorname{germ}(g)$.

Note that $\operatorname{germ}(k)=k$ for $k \in K$.

Proof. We prove this by induction on $|g|$. Write

$$
g=\left\langle\left\langle g_{0}=x, \ldots, g_{m^{n}-1}\right\rangle\right\rangle \tau
$$

for the decomposition of $g$ at level $n$. Assume that the claim holds for $g$, i.e., $x \in K$; we will show it for $g s$ where $s$ is a generator. Since $x \in K$, we have $0 . x=0$ and the first-level section satisfies $x(0)=x$. Then for a generator $s=\left\langle\left\langle s_{0}, \ldots s_{m^{n}-1}\right\rangle\right\rangle \sigma$ we have

$$
g s=\langle\langle x y, \ldots\rangle\rangle \tau \sigma
$$

where $y=s_{0 . \tau}$ is itself a generator or the identity. At level $n+1$ we have the expansion

$$
g s=\langle\langle x y(0), \ldots\rangle\rangle \tau^{\prime} \sigma^{\prime} .
$$

Now for every generator $y$ if $y \notin K$, then $y(0)=\mathrm{id}$. In this case we have $x y(0)=$ $x(0)=x$, and the level $n+1$ expansion is of the form $g s=\langle\langle x, \ldots\rangle\rangle \tau^{\prime} \sigma^{\prime}$. Note that $x(0)=x$ implies that the level sections of $g$ at the zero ray remain $x$ from level $n+1$ on.

Otherwise, if $y \in K$, note that $0 \cdot x=0$, and $y(0)=y$, and therefore $x y(0)=$ $x(0) y(0 \cdot x)=x y$, so the level sections of $g$ remain $x y$ from level $n$ on, the completing the proof. 
We define $\operatorname{lamp}(g)$ as the quotient of germ $(g)$ by the subgroup group generated by all generators of degree at most $d-1$. The function lamp( ) takes values in the coset space $K / H$.

Proposition 14 (Lighting a single lamp). Let $g \in M$ and let $s$ be a generator. Then we have

$$
\operatorname{lamp}(g s)= \begin{cases}\operatorname{lamp}(g) \operatorname{lamp}(s) & \text { if } g \text { fixes the zero ray, } \\ \operatorname{lamp}(g) & \text { otherwise. }\end{cases}
$$

Proof. We use the notation of the previous proof. The proof implies

$$
\operatorname{germ}(g s)=\operatorname{germ}(g) \operatorname{germ}(y)
$$

(for the generator $y$, if $y \notin K$ then $\operatorname{germ}(y)=$ id).

If $g$ fixes the zero ray, then $0 . \tau=0$ and $y=s$, so the claim follows from (5).

If $g$ does not fix the zero ray, then at a high enough level $0 . \tau \neq \tau$. Working on a level where this happens and also the zero-ray section of $g$ is stable, we get $y=s_{0 . \tau}$, and therefore $y$ is of lower degree than $s$. Thus $\operatorname{lamp}(y)=\operatorname{id} H$ (trivial), and the claim follows from (5).

We are now ready to prove Theorem 3, which states that if the Schreier graph of the mother group the zero ray is transient, then no non-degenerate random walk with finitely supported step distribution has the Liouville property.

Proof of Theorem 3. Note that transience is quasi-isometry invariant, and changing generators is a quasi-isometry of the Schreier graph. Thus the Schreier graph for the random walk $Y_{1} \ldots Y_{n}$ in question is also transient. Thus $Y_{1} \ldots Y_{n}$ in question fixes the zero ray only finitely many times. In particular, $\operatorname{lamp}\left(Y_{1} \ldots Y_{n}\right)$ stabilizes at some random value $L$ with probability 1 .

We now claim that $L$ takes all possible value in $K / H$ with positive probability, and hence the Poisson boundary is nontrivial. Indeed, let $l \in K / H$ with $|l|=n$. Then with positive probability $Y_{1} \ldots Y_{n}=l$. Moreover, by transience of the Schreier graph, the independent walk $Y_{n+1} \ldots Y_{n+k}, k \geq 0$ never fixes the zero ray for $k \geq 1$ with positive probability. In this case $L=\ell$.

We have shown that the Poisson boundary is nontrivial. Now, by the standard construction, function $x \mapsto P_{x}(L=\mathrm{id})$, where $x$ is the starting point of the random walk, is bounded, harmonic and nonconstant.

Acknowledgements. Part of this work was done while the first author was at the University of Toronto. The work of the second author was supported by the Canada Research Chair program and the NSERC Discovery Accelerator program. In addition, he thanks the Department of Stochastics, Technical University of Budapest where this work was completed. 


\section{References}

[1] G. Amir, O. Angel, and B. Virág, Amenability of linear-activity automaton groups. J. Eur. Math. Soc. (JEMS) 15 (2013), 705-730. Zbl 1277.37019 MR 3085088

[2] L. Bartholdi, V. A. Kaimanovich, and V. V. Nekrashevych, On amenability of automata groups. Duke Math. J. 154 (2010), 575-598. Zbl 1268.20026 MR 2730578

[3] L. Bartholdi and B. Virág, Amenability via random walks. Duke Math. J. 130 (2005), 39-56. Zbl 1104.43002 MR 2176547

[4] I. Benjamini and C. Hoffman, $\omega$-periodic graphs. Electron. J. Combin. 12 (2005), Research Paper 46. Zbl 1098.05069 MR 2176522

[5] A. Erschler, Boundary behavior for groups of subexponential growth. Ann. of Math. (2) 160 (2004), 1183-1210. Zbl 1089.20025 MR 2144977

[6] R. I. Grigorchuk and A. Żuk, Spectral properties of a torsion-free weakly branch group defined by a three state automaton. In Computational and statistical group theory (Las Vegas, NV/Hoboken, NJ, 2001), Contemp. Math. 298, Amer. Math. Soc., Providence, RI, 2002, 57-82. Zbl 1057.60045 MR 1929716

[7] V. A. Kămanovich and A. M. Vershik, Random walks on discrete groups: boundary and entropy. Ann. Probab. 11 (1983), 457-490. Zbl 0641.60009 MR 704539

[8] V. Nekrashevych and A. Teplyaev, Groups and analysis on fractals. In Analysis on graphs and its applications, Proc. Sympos. Pure Math. 77, Amer. Math. Soc., Providence, RI, 2008, 143-180. Zbl 1162.28004 MR 2459868

[9] G. Pete, Probability and geometry on groups. Lecture notes, 2009. http://www.math.bme.hu/ gabor/PGG.html

[10] S. Sidki, Automorphisms of one-rooted trees: growth, circuit structure, and acyclicity. $J$. Math. Sci. 100 (2000), 1925-1943. Zbl 1069.20504 MR 1774362

[11] N. T. Varopoulos, Long range estimates for Markov chains. Bull. Sci. Math. (2) 109 (1985), 225-252. Zbl 0583.60063 MR 822826

[12] A. M. Vershik, Dynamic theory of growth in groups: entropy, boundaries, examples. Uspekhi Mat. Nauk 55 (2000), 59-128; English transl. Russian Math. Surveys 55 (2000), 667-733. Zbl 0991.37005 MR 1786730

Received February 28, 2011; revised August 8, 2012

G. Amir, Department of Mathematics, Bar Ilan University, Ramat Gan, 52900, Israel

E-mail: amirgi@macs.biu.ac.il

B. Virág, Departments of Mathematics, University of Toronto, Toronto ON M5S 2E4, Canada

E-mail: balint@math.toronto.edu 